\title{
The Deft-FEWS flood forecasting system and its application in the middle-upper parts of the Huaihe River Basin, China
}

\author{
Kai Wang, Mingkai Qian, Shunfeng Peng, Shijin \\ $\mathrm{Xu}$, Fengsheng Li \\ Hydrologic Bureau of the Huaihe River Commission, \\ the Ministry of Water Resources \\ Bengbu, China \\ e-mail: wangkai@hrc.gov.cn
}

\author{
Min Xu \\ Operational Water Management Unit, Deltares \\ Deltares \\ Delft, 2600MH, the Netherlands \\ e-mail:min.xu@deltares.nl
}

\begin{abstract}
In order to solve the problems of multi-sources data management and multiple models integration, this study employs open source system-Delft-FEWS developed by Deltares in the Netherlands, to conduct flood forecasting in the Huaihe River Basin of China for the first time. The system provides a platform for data handling and is open to connect a wide range of monitored and forecasted weather inputs on the one side and of hydrological and hydraulic models on the other side. The application shows that Delft-FEWS is a useful and efficient tool for flood forecasting in the Huaihe River Basin, China. Particularly, as an open system - joint development approach, Delft FEWS shows a promising potential for application as a open flood forecasting platform in the Huaihe River Basin, which will facilitate probability flood forecasting for the Huaihe River Basin.
\end{abstract}

Keywords-Flood forecast, Deft-FEWS, open source and open shell, Huai River Basin, China

\section{INTRODUCTION}

Flood forecasting is crucial for flood prevention and disaster reduction. However, traditionally most flood forecasting systems have been built around the application of hydrological and/or hydraulic routing models. This modelcentric approach often tailor made to suit situation. However, it is inflexible to changing model needs and data availability. With the increasing availability of weather forecast data, including numerical weather prediction, Radar data, on-line observations (precipitation, discharge etc.), high resolution global geographical data sets, and with the evolution of state of the art modeling, challenges are not the modelling but efficient handling of large datasets and flexible and open system to enable easy model integration.

In response to these challenges, Delft-FEWS provides a state of the art hydrological forecast and warning system. The system is a sophisticated collection of modules designed for building a hydrological forecasting system customized to the specific requirements of an individual organization. The philosophy of the system is to provide an open shell for managing the data handling and forecasting process. This shell incorporates a comprehensive library of general data handling utilities, allowing a wide range of external forecasting models to be integrated in the system through a published open interface. There have been many successful applications of Delft-FEWS over the world from national level to provincial level, more details can be found in the references $[1,2]$. In this study, Delft-FEWS is applied to the middle-upper parts of the Huaihe River basin in China. The study is at the beginning stage and two sub-systems are considered for both hydrological and hydraulic processes.

The paper is organized as follows: Section II provides an overview of the open data management and model integration platform-Deft-FEWS, with special regard to its openness and the key to open model integration-model adapter; Section III applies the Deft-FEWS in the pilot site of the Huaihe River Basin for testing the feasibility and potential for application in the Huaihe River Basin. Section IV finally provides a summary of the paper, as well as an outlook on the future development of Delft-FEWS in the Huaihe River Basin.

\section{AN OPEN FLOOD FORECASTING SYSTEM: DEFT-FEWS}

The Deft-FEWS was developed with the aid of JavaTM and J2EE. It is an independent platform that can be run in both Windows and Linux, and has independent database that can link to Oracle, Postgres and SQL-Server, etc. It can be used in standalone and in automated server/client modes.

\section{A. Philosophy of Deft-FEWS}

The Deft-FEWS has been developed as a data management and model integration platform. On one hand, this platform is open to various data sources supplying measured or forecasted weather state variables, such as precipitation and temperature. On the other hand the platform enables the generic coupling of a variety of hydrological/hydraulic models (Fig. 1). It create links to all kinds of meteorological data sources, such as ground station monitoring devices, weather radar, numerical weather models and satellite images and provide facilities to process such data in required formats. The data management platform of the Deft-FEWS has been equipped with generic tools providing a variety of data handling tasks, such as data validation, interpolation, aggregation and error correction in forecasts, including a variety of visualization and forecast dissemination options. It also create links to a wide range of hydrological and hydraulic modelling systems, such as HBV, Sacramento, Sobek, and Mike11, etc. The most important is 


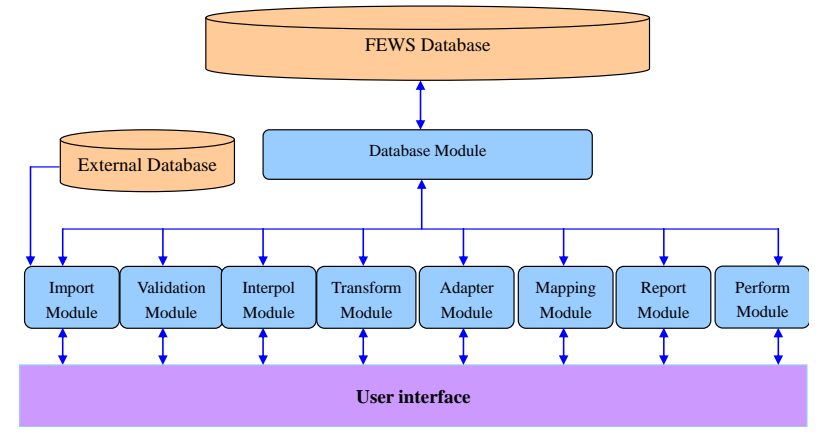

Figure 1. The Delft-FEWS modular structure.

that it is easy to connect all kinds of models and data sources[3] There have been many successful applications of Delft-FEWS over the world from national level to provincial level,more details can be found in the references $[1,2]$.

\section{B. Powerful Data Management Capability of Deft-FEWS}

\section{1) Data import and storage}

In most operational systems, data from several sources is considered, with different data networks typically using different formats for storing and publishing data [4]. Efficient import of data from these different sources poses a significant challenge, not only due to the variety of formats being used, but in many cases also due to differences in the meta-data provided.

In response to these challenges, Delft-FEWS provides a data import module that has been designed to handle a wide range of data formats [5]. It provides standard import modules to import data from several on-line external hydrological and meteorological data sources. The import of external data also supports ensemble weather predictions. Data is preferably imported using standard interchange formats, such as XML, GRIB (for Numerical Weather Predictions) and ASCII, but alternative import procedures are also available. Manual data entry is also possible though the data editor. If data quality information is available, DelftFEWS can also interpret and import this information. Imported data may for example include time series obtained from telemetry systems such as observed water levels, observed precipitation, but also meteorological forecast data, radar precipitation data, etc.

All data is stored within the Delft-FEWS database. This contains both configuration data, including location specific information (e.g. coordinates and properties of forecasting points, map layers etc.), and dynamic time series data either imported from external sources. Dynamic data can additionally be produced by internal data manipulation methods or models run from within the system [5]

2) Data validation, interpolation and transformation

Particular emphasis is placed in Delft-FEWS on quality control of data. Through a step-wise process of successive processing steps the data that is eventually provided to a forecast model is ensured to be of the best possible quality. This data-centered approach is the backbone of Delft-FEWS.
Three Delft-FEWS modules, namely data validation, data interpolation and data transform cover all the required processing steps.

Data validation and serial and spatial interpolation of incoming data in FEWS use extensive data validation rules, including user-defined validation rules. Readily available interpolation methods are regression functions, Kriging, Thiessen polygons and the inverse distance method; Options with gap-filling and hierarchy rules allow alternative data sources to be used as a fall-back to ensure continuity in the forecasting process.

Another set of utilities is available for transforming data with disparate spatial and temporal scales. This includes spatial interpolation to derive for example areal weighted precipitation from spatially distributed point sources, or from spatial data such as radar data and numerical weather prediction models. Data transformation utilities also include methods for temporal aggregation and dis-aggregation, evaluation of simple equations, and typical hydrological functions such as stage-discharge relationships and evaporation calculations [6].

3) Open integration of external model in Deft-FEWS

Delft-FEWS provide an open system that allows a wide range of existing forecasting models to be used. This concept is supported by the general adapter module, which communicates to external modules through an open XML based published interface, effectively allowing "plugging-in" of practically any forecasting model.

An adapter between the native module data formats and the open XML interface is typically required, and such adapters are already available to support a wide range of hydraulic and hydrological models. The great advantage of this open interface is that existing hydrological and hydraulic models and modeling capabilities can easily be integrated in the forecasting system, without the need for expensive remodeling using a specific model. What follows will further discuss the core of the open integration approach -model adapter.

In a model adapter the following activities are taking place (Fig. 2):

- data from FEWS (in PI-XML format) is converted into 'native' model input. This can be time series, parameter information, state information etc. CSV format file as a native format was adopted in this study.

- the model itself is switched on and runs without any user interaction (=headless)

- native output from the model (time series, states, logging etc.) is converted back into readable format for FEWS (=PI XML).

Exporting data to PI-XML (prior to step 1) and importing it back into FEWS (after step 3 ) is taken care of by a specific module of Delft-FEWS called the General Adapter. This General Adapter is part of the FEWS code. The General Adapter and the Model Adapter are closely related. The General Adapter calls the Model Adapter to do its job as part of the complete modelling workflow. 


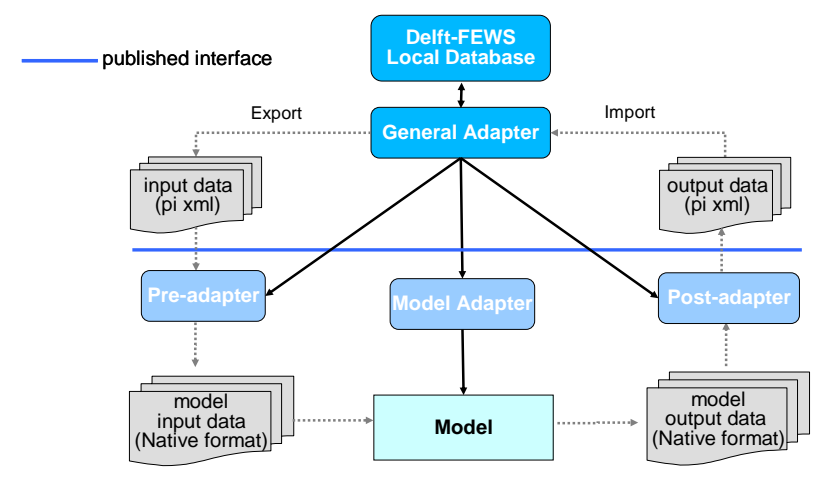

Figure 2. The Open communication between Delft FEWS and Models through published interface.

A model adapter can be developed in different development languages (Java, C\#, VB). There are numerous existing libraries shared by Deltares [6], however, a model adapter is needed for newly integrated models in Deft-FEWS. The details of model adapter for Deft-FEWS's application in the Huaihe River Basin will be discussed in the following section.

\section{APPLICATION OF DEFT-FEWS IN THE HUAIHE RIVER BASIN-FEWS HUAIHE}

\section{A. Study Areas}

Huaihe River basin is located in eastern China between the Yellow River and the Yangtze River, shown in Fig. 3. The whole basin has a total area of $270,000 \mathrm{~km}^{2}$, covers five provinces and can be divided into several sub-catchments, with a length of 1,000 kilometers. The average annual precipitation is $875 \mathrm{~mm}$.

The Huaihe River Basin has the following major features. (1)The Huaihe River Basin is located in the transitional zone from southern to northern climate of China, the weather system changes dramatically, inter-annual precipitation varies greatly and spatial-temporal distribution of intraannual precipitation is also uneven. Particularly, there are two types of rainstorms in the Huaihe River basin. The first one is the plum rain with characteristics of extensive coverage, large amount and long duration. This type of rain can easily cause basin-wide disaster. The second type is the typhoon rain with characteristics of high intensity and short duration, which can easily cause local disaster [7]. (2)Historically, with the diversion (capture) of the headwaters of Yellow river into the Huaihe River, the natural water system in the middle-low reaches changes dramatically. (3)The Huaihe River Basin has a broad plain area (accounts for $2 / 3$ of the total area), which has poor drainage condition and relatively small storage capacity [8]. Therefore, flood frequently hits the Huaihe River Basin. In order to mitigate flood damage, proper flood forecasting is urgently required.

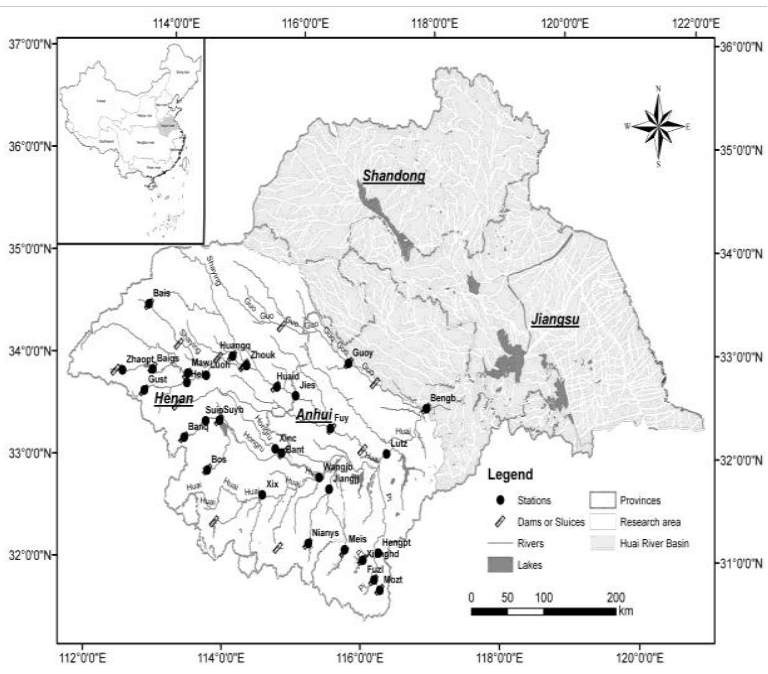

Figure 3. Map of the Huai River Basin.

The upper part and middle reach of the Huaihe River are selected for the study. These two systems have different characteristics. The upper part is a hydrological basin, located at the south-west of the Huaihe River basin. It is the upstream part of the basin with steep geological slope, which can generate fast runoff. The middle reach of the Huaihe River is a hydraulic system of open channel flow from Zhengyangguan to Bengbu. This region is relatively flat and flooding can easily occur. Therefore, retention areas have been created beside the river to divert flood peaks, together with lakes.

\section{B. Data Import, Storage and Manipulation}

A wide range of data has been imported in FEWSHUAIHE in this study, such as vector data (drainage map, map of rainfall station, discharge station etc.), gridded data (Global Forecast System data from USA) and scalar data (Precipitation, discharge, water level and evaporation), etc. The rainfall data in practice is recorded every 1 hour or 6 hours in the database. However, the model uses a 2-hour time step.

In this study, data disaggregation, merge, aggregation and data validation were employed to manipulate data. The rainfall data in practice is recorded every 1 hour or 6 hours in the database. However, the model uses a 2-hour time step. Delft-FEWS uses the functions of disaggregation, merge and aggregation to process the data. 6-hour data is disaggregated to 1 hour. Together with the hourly recorded data, the merge function selected the time series based on their hierarchy. For example, when hourly data are not available but 6-hour data are available in the database, the disaggregated hourly data is used instead. Finally the 2-hour data is generated by aggregating the hourly data. Fig. 4 shows data import and manipulation in FEWS-Huaihe. 
TABLE I. HYDROLOGICAL AND HYDRAULIC MODELS USED IN THE HUAIHE RIVER BASIN AND RELEVANT MODEL

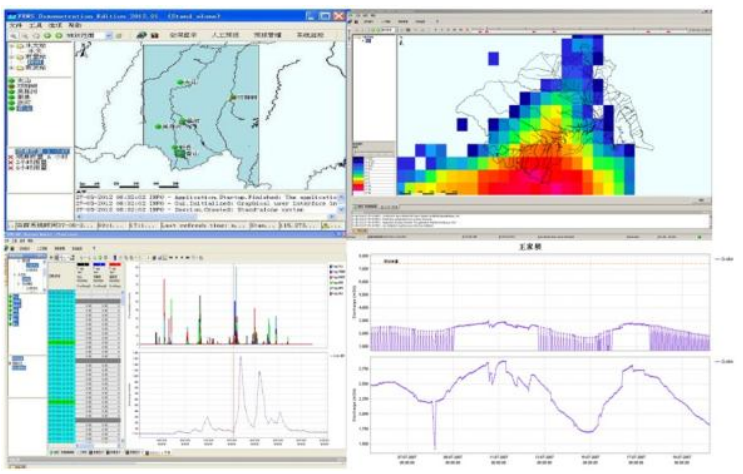

Figure 4. Data import and manipulation in FEWS-Huaihe (Vector data on top-left, gridded data on top-right and scalar data at the bottom and data manipulation at bottom-right).

\section{Integration Of Hydrologic And Hydraulic Models}

In Huaihe River basin, numerous hydrological and hydraulic models are used for flood forecasting, such as Xinanjiang model, tank, API empirical model and unit hydrograph for hydrological forecasting, and HEC-RAS and empirical models for hydraulic forecasting. Xinanjiang model [9] and HRC-RAS model [10] are the two implemented in this study. Because this study is intended to introduce the capability of Delft-FEWS integrating multiple models \& multiple data sources and facilitate flood forecasting, the detailed model description is not the focus. What follows will briefly introduce the hydrological and hydraulic models used in the Huaihe flood forecasting, focusing on development of model adapters and demonstrate integration of external models into Deft-FEWS by model adapter.

\section{1) Hydrologic and hydraulic models}

In Huaihe River basin, several hydrological and hydraulic models are used for flood forecasting, such as Xinanjiang model, tank, API empirical model and unit hydrograph for hydrological forecasting, and HEC-RAS, SOBEK and empirical models for hydraulic modeling (Tab. 1). The detailed model description will not be discussed because this study is intended to introduce the capability and potential of Delft-FEWS integrating multiple models \& data sources and facilitate flood forecasting.

\section{2) Model adapter development}

Model adapters are the core of external model integration in Deft-FEWS. What follows will discuss the model adapter development in the FEWS system for the Huaihe River Basin.

a) Model adapters for models not existed in DeftFEWS.

Model execution in Delft-FEWS is triggered through a general adapter, of which the process is illustrated in Fig. 3. The process involves two components: a general adapter and a model adapter. General adapter converts data from the Delft-FEWS database into PI XML data format.

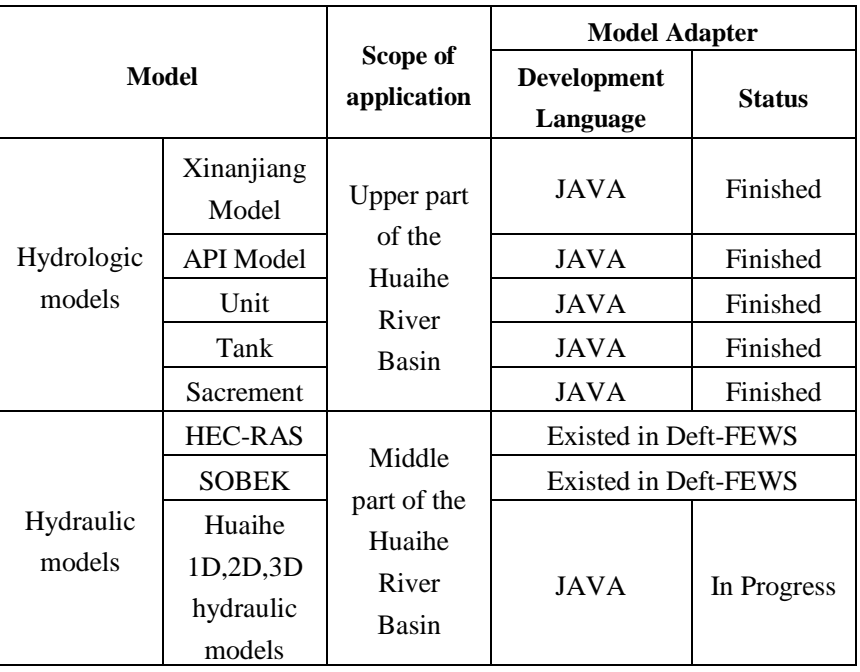

Model adapter contains a pre-adapter and a post-adapter. The pre-adapter converts the XML files generated by the general adapter to the model input format (Native format). Once the input data are ready, the model adapter triggers the model run. After the model simulation is completed, the post-adater converts the output data (Native format) back into XML data format and import them into the Delft-FEWS database.

The model adapter for FEWS-Huaihe was developed using Java, including a pre-adapter, a post adapter and TimeSeriesParser class. The hydrologic models from the Huaihe River Commission was developed with Visual Basic 6.0, and input data (parameter data, precipitation data, state information) using the CSV format. With the help of Preadapter, input data (XML format) from FEWS can be converted to CSV format to feed the Hydrologic models. On the other hand, with the aid of Post-adapter, output (CSV format) from Hydrologic model can be converted to XML format to be interpreted by the FEWS.

b) Model adapters for existing models in Deft-FEWS.

As mentioned previously, HEC-RAS and SOBEK are employed in flood forecasting for the middle part the Huaihe River Basin. Deft-FEWS has been widely used all over the world, and numerous models adapters are available for existed hydrologic models and hydraulic models, such as model adapters for HEC-RAS, SOBEK and MIKE. Therefore, in this study, model adapters for the existed models (HEC-RAS) and SOBEK are available, only a few minor configurations are needed for model integration [6].

\section{RESULTS OF THE FEWS-HUAIHE}

The Xinanjing and HEC-RAS models are applied to the upper and middle parts of the Huaihe River Basin, and the results are shown in Fig. 6. The lower curve is result of Xinanjiang model and the top curve represents HEC-RAS output. In the figure, the vertical red line represents the current time T0. Left side of T0 is the historical data and simulation, and the right side represents forecasting. As shown in the result of the Xinanjing model at the bottom of 


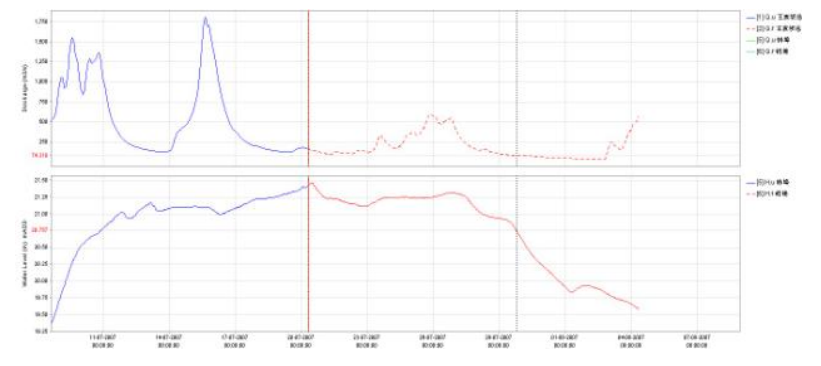

Figure 5. FEWS-Huaihe outputs (Xinanjiang model for the upper part shown at the bottom and HEC-RAS for the middle part shown on the top).

the figure, lower part of the curve shows the simulated runoff. It is noticed that the model can capture all the rain events, and there exists delays of a couple of hours from rainfall to runoff. The upper part is a steep slope area and the delay time is also in a logic magnitude. The Hec-Ras results are also reasonable compared with historical data.

\section{CONCLUDING REMARKS}

The present article gives a brief introduction of DelftFEWS for serving operational flood forecasting. The platform facilitates the integration of meteorological forecasting systems with hydrological as well as hydraulic models within an open forecasting environment.

The principal advantage of this approach is that it enables a shift from a model-centred forecasting approach towards a data-centred system. In this approach, already existing software modules and models such as hydrological, hydraulic can be mutually combined, re-used and encapsulated into (new) forecasting environments, whereby the data organization remains unchanged. Examples are the Xinanjiang and HEC-RAS models already in place in the Huaihe River Basin. The successful application shows the capability of Delft-FEWS to integrate multiple models into a complete system and facilitate forecasting for an organization. In addition, Delft-FEWS has a large amount of build-in functions to process data which makes data management convenient for users.

In the future the FEWS Huaihe will be extended to all the river basins, with more hydrologic and hydraulic models, such as SOBEK, Tank, API empirical model etc. Other developments include technical improvements. As an example the use of radar data is still troublesome due to accuracy as well as data sharing issues. An interesting future option for FEWS-Huaihe is the use of weather forecast from numerical weather prediction models. At the moment no output of weather prediction models is used in FEWSHuaihe, but some Delft-FEWS applications in Europe (like FEWS NL in the Netherlands) heavily depend on the use of weather prediction models. With the integration of weather prediction and numerous meteorological data sources, another promising potential is to employ uncertainty analysis in Deft-FEWS for operation probability flood forecasting in Huaihe River Basin.

\section{ACKNOWLEDGMENT}

The study is supported by Non-profit Industry Financial Program of Ministry of Water Resources of China (201301066) and Rijkswaterstaat of Netherlands.

\section{REFERENCES}

[1] Werner, M. and Heynert, K., 2006. 2006. Open Model integration - a review of practical examples in operational flood forecasting. Proceeding of the 7th International Conference on Hydroinformatics, Gourbesville, Cunge, Guinot and Liong (eds), Research Publishing, Vol.1, 155-162.

[2] Schellekens, J., Weerts, A.H., Moore, R.J., Pierce, C.E. and Hildon, S., 2011. The use of MOGREPS ensemble rainfall forecasts in operational flood forecasting systems across England and Wales, Advances in Geosciences, 2011, 29, 77-84..

[3] Adri Verwey, Karel Heynert, Micha Werner, Paolo Reggiani, Bob van Kappel and JanJaap, 2006. The potential of the delft-fews flood forecasting platform for application in the mekong basin, Proceeding of the 4th Annual Mekong Flood Forum, Siem Reap, Cambodia, 18 19 May 2006. pp. 110-115.

[4] Horsburgh, J.S., Tarboton, D.G., Piasecki, M., Maidment, D.R., Zaslavsky, I.,Valentine, D., Whitenack, T., 2009. An integrated system for publishing environmental observations data. Environmental Modelling \& Software 24, 879-888.

[5] Werner, M., Schellekens, J., Gijsbers, P., van Dijk, M., van den Akker, O. and Heynert, K., 2012. The Delft-FEWS flow forecasting system, Environmental modeling \& software, http://dx.doi.org/10.1016/j.envsoft.2012.07.010.

[6] Deltares, 2010, Deft-FEWS software, http://www.deltares.nl/en..

[7] Wang B., 2010. The 2007 Huaihe River storms and floods, China Water Publication, 1-25.

[8] Qian, M. , 1992. Handbook of water issues in the Huai River Basin, China Water Publication, 1-10.

[9] Zhao, R. J., Zhuang, Y. L., Fang, L. R., Liu, X. R., and Zhang, Q. S., 1980. The Xinanjiang model. The XinanjiangModel, Hydrological Forecasting Proceedings Oxford Symposium, Wallingford, U.K., 129, p. 351-356.

[10] U.S. Army Corps Engineers, 2010. HEC-RAS River Analysis System Hydraulic Reference Manual version 4.1, www.hec.usace.army.mil. 